\title{
Research on the Development Problems and Countermeasures of Lei Opera
}

\author{
Li chunfei

\begin{abstract}
Department of music, Nan fang College of Sun Yat-sen University, Guangzhou, Guangdong,510000,China
\end{abstract} \\ *Corresponding author's e-mail: 861801953@qq.com
}

\begin{abstract}
The development of Lei opera (the locai drama in Lei Zhou Peninsula in Guangdong) has experienced four stages: "Guniang song (Girl song) ", " Quanshi song(Exhortation song) ", "Daban song (Big class song) " and "Lei opera".Although it has a history of 500 years, it is still difficult to get out of Lei Zhou dialect area. This paper analyzes the development status of Lei opera through field investigation and analysis, and finds that the development of Lei opera is difficult to spread and develops slowly due to the lack of innovation, shortage of talents, loose market supervision, single transmission channels and lack of academic research. Facing this question, the author puts forward some corrective suggestion and countermeasure about it, in order to arouse the attention of the relevant cultural departments.
\end{abstract}

Keywords: Lei opera, Leizhou culture, development status, countermeasure research

\section{INTRODUCTION}

Lei opera is one of the four Han operas in Guangdong Province. It originated from the folk song of Leizhou Peninsula - Leizhou song. It has more than 70 tones and has profound cultural background and humanistic value. Due to dialect limitations, Lei opera is mainly popular in Leizhou dialect areas, such as Leizhou City, Xuwen County, Suixi County, and Dianbai County. It is especially active in Leizhou City. Almost every independent small village will hold an annual live performance for Lei opera, usually about 10-30 days, which is deeply loved by the public.

At present, Lei opera has gradually stepped out of Leizhou Peninsula and achieved outstanding results, which has historical significance: in 1992, the opera "Zhuajiu village head" won the first " Five "One" Project " award of Publicity department of the Communist Party of China central committee; in 2001, Lin Fen, the protagonist of opera " Liang hongyu be marshal ", won the " Plum Blossom Prize" of the highest award of Chinese opera; in 2010, Lei opera was listed in the national intangible cultural heritage list. Although Lei opera has a remarkable artistic value, its current development status is not satisfactory, and the relevant issues deserve our attention and research.

\section{THE PROBLEM IN THE DEVELOPMENT OF LEI OPERA}

\subsection{Lack of innovation}

Lei opera's lyrics are formalistic and obscure, and the themes are mostly from historical allusions. They are rich in content but old and single, and the story plot lacks new ideas. They fail to meet the theme of the times and keep pace with the times. The audiences are familiar with the traditional plays that have not changed for a hundred years, and their enthusiasm for watching them will naturally be reduced.

\subsection{Lack of talents}

The lack of talents is the biggest bottleneck of the development of Lei opera, the scarcity of performing talent and creative talent is particularly prominent. At the beginning of the 21st century, Leizhou city vocational and technical school offers free Lei opera class for participants. However, due to the low status of Lei opera actors in people's traditional concepts, the difficulty of learning and the low economic income of engaging in related work, many parents love Lei opera but do not support their children to engage in related jobs, so the enthusiasm of the younger generation to learn Lei opera has not been greatly improved and it is difficult to solve the problem of lack of talents in Lei opera.

\subsection{The artistic level of the troupe is uneven}

The existing Lei opera troupes have different artistic levels and organizational systems. The outstanding representative Lei troupe is like Zhanjiang experimental Lei troupe, which won the most awards. The troupe has perfect mechanism and strict management, exquisite art level, and is deeply loved by the audience. However, due to the low entry threshold of Lei opera actors, the management and assessment mechanism of the troupe for the actors is not perfect, especially for the amateur Lei Opera Troupe, many actors are directly learning from the troupe without professional and systematic training, resulting in rough performance and 


\section{COUNTERMEASURES TO PROMOTE THE DEVELOPMENT OF LEI OPERA}

\subsection{Loose market supervision}

Government departments have loose supervision over the market of Lei opera, lack of laws and regulations related to Lei opera, and the development of opera troupes is almost unregulated. Vicious market grabbing phenomena such as driving up or lowering prices occur from time to time. Many troupes are in a state of loss all the year round due to poor management and are faced with the risk of dissolution.

\subsection{The way of communication is single and the audience group is aging seriously.}

The main way of communication of Lei opera is to sing on the spot.At present, resources related to Lei opera are extremely scarce on the platforms such as: Popular short video player, WeChat Official Account, Film and television player, and music player etc. And it's basically recorded by individual Lei opera lovers, with problems such as uneven artistic level and poor recording effect and limited resources. The way of communication of Lei opera directly affects the width and breadth of the audience. At present, the aging of the audience is becoming more and more serious. The young generation has little chance to contact Lei opera, which leads to the low attention and influence of Lei opera in the youth group, and the loss of interest in Lei opera over time.

\subsection{Lack of academic research}

In the middle of the 20th century, in order to speed up the reform and development of Lei opera, Zhanjiang city actively mobilize writers of Lei opera to create and compile books related to Lei opera. The existing works include "Lei Playbook compiled " by Song Rui, Chen Xiang and Zhan Nansheng, " L ei Yun ", " stage sentiment ", " selected aria of Lei opera " and " Lei opera music " of Chen Xiang. " Lei opera " of Chen Zhijian and "LeiGe daquan " of Lin Tao All of them; " collected papers " of hundred years' Lei opera forum in Leizhou City; " cultural records " of Zhanjiang City compiled by Zhanjiang Culture Bureau; " Zhanjiang art compiled "by Zhanjiang Art Research Office, etc., but at present, there is a lack of literature about Lei opera which can be found on China Knowledge Network, e-book reading software and market. First, there is a lack of relevant achievements; and second, some books such as Chen Hunan's "Lei Yun", "stage sentiment", "selected aria of Lei opera", "music of Lei opera" cannot be consulted and purchased due to the lack of relevant information and out of print on the internet.

\subsection{Actively promote the reform and innovation of Lei opera}

The government should pay more attention to the reform and innovation of Lei opera, and actively promote the new aria. At the same time, we should pay attention to the cultivation of the creative spirit and ability of the creators of Lei opera. Encourage the bold application of new aria for creation, pay attention to the reform of lyrics, and avoid the situation where the use of words is rigid and far fetched for the sake of symmetry and rhyme. We should keep up with the pace of the times, integrate new themes, enrich the story, and innovate on the basis of inheritance, which will Make a deep impression on the audience and increase their interest in watching the play.

\subsection{Pay attention to personnel training and assessment}

Regarding the cultivation of reserve talents, the propaganda of lei opera culture should be strengthened, and effective measures should be taken, such as creating and playing the role of idols, to improve the social status of lei opera workers.We will explore more scientific and effective teaching methods, increase financial support and reasonably raise the salary of relevant jobs, so as to attract more people to join the career of Lei opera.

For the existing actors, the daily management should have a strict assessment system. Government departments and troupes should pay attention to the continuous training and assessment of actors, organize regular training of actors, provide them with a good development platform and learning opportunities, strictly grasp the professional quality of actors, and ensure the performance quality.

\subsection{Improve the management mechanism of the troupe}

The government should improve the management mechanism of the theater, conduct regular assessment of actors and evaluation of the theater, and promote the orderly development of the theater. Practically improve and strictly implement the entry standards of the troupe and performers. Before taking the post, the actors must learn the relevant theoretical knowledge and performance skills, pass the qualification examination, and take the post with the certificate; the troupe business must pass the professional evaluation including the actor's qualification, member structure, hardware equipment and other aspects before obtaining the business license. 
Mall, Amazon and other websites for sales.

\subsection{Attach importance to the construction of legal system and strengthen the macro-control of the market by the government}

On the basis of fully respecting the law of development of Lei opera in combination with the actual situation,as well as,relevant laws and regulations should be promulgated,the market economy system of Lei opera should be improved.The market of Lei opera performance should be governed according to law, and unlicensed opera troupes and vicious market grabbing behaviors should be resolutely banned.

\subsection{Make full use of network platform to promote the propagation speed of Lei Opera}

Under the background of the information age, it is not enough to rely on the original on-site viewing mode for the dissemination of Lei opera. We should establish a special cultural department to promote the diversified channels of the dissemination of Lei opera. Make full use of the network platform to record and upload the performance video and audio of Lei opera. Uploading the complete video of Lei opera on the film and television broadcasting platform, live broadcasting the live performance of Lei opera in real time on the live platform, making the wonderful clips into small videos, etc. can enable young people to have more channels to enjoy Lei opera, improve the aging problem of Lei opera audience groups, and help Lei opera "go out".

\subsection{Pay attention to the academic research of Lei opera and should be done well in sharing achievements}

We should strengthen the academic research of Lei opera, vigorously mobilize the cooperation and exchange between scholars from all walks of life and Lei opera actors, and increase the government's financial support for academic research to promote the output of research results. Scholars from all walks of life have solid theoretical basis and scientific research methods, but there may be deviation in understanding and understanding of Lei opera; Lei opera actors are front-line workers in the development of Lei opera, with rich practical experience but lack of theoretical guidance, so it is difficult to independently complete in-depth academic research. Only when the two sides work together to explore the mode of scientific inheritance and reform can we achieve the most win-win situation.

For the problem of hard to find relevant materials, the cultural department should be done well in the publicity, sharing and protection of results. Research papers should be published in journals included in knowledge platforms such as HowNet. Monographs and literature should be printed according to market demand. In addition to physical bookstores, they should also be widely distributed in major e-commerce platforms such as dangdang.com, Jingdong
In addition to theoretical research, recording teaching videos of Lei opera masters for permanent preservation for learning and research is an effective method of inheritance.

\section{CONCLUSION}

Lei opera is a unique cultural card of Lei Zhou Peninsula. It is a cultural and artistic treasure reflecting the local conditions and customs of one side and the common good wishes of the people. Its development is faced with many challenges but full of opportunities at the same time. It is hoped that the government departments can pay more attention to relevant issues and intervene them, so as to open up a broader development path for the development of Lei opera.

\section{REFERENCES}

[1] Chen zhijian, Lei Opera, Guangzhou People's Publishing House, Guangdong. (2010)

[2] Bu chunkai, Zheng xiaoye, Wang kangyong, Analysis of The Development Status and Strategies of Lei Zhou Peninsula Lei Opera [J],Literature Education (ii).(10)(2019) , pp. 152-154.

[3] Liu xiankun, Research on The Inheritance and Development of Lei Opera of Zhanjiang's National Intangible Cultural Heritage, Master's thesis of Guangdong ocean university. (2018)

[4] Chen yaolong, The Ritual and Music of Matsu's Fiesta in“Nian li"Custom(Wenzhangwan Village,Zhanjiang City), Master's thesis of Chinese National Academy of Arts. (2014)

[5] Lin wei, Investigation and Research on the Education of Lei Drama Class in Leizhou Vocational-Technical School, Master's thesis of Guangzhou university. (2017) 